\title{
XI. New experiments relative to the action of magnetism on electro-dynamic spirals, and a description of a new electromotive battery
}

\author{
Signor Salvatore Dal Negro \& Michael Faraday Esq. F.R.S. M.R.I.
}

To cite this article: Signor Salvatore Dal Negro \& Michael Faraday Esq. F.R.S. M.R.I. (1832) $\mathrm{XI}$. New experiments relative to the action of magnetism on electro-dynamic spirals, and a description of a new electromotive battery , Philosophical Magazine Series 3, 1:1, 45-49, DOI: $10.1080 / 14786443208647818$

To link to this article: http://dx.doi.org/10.1080/14786443208647818

Published online: 01 Jun 2009.

Submit your article to this journal $\sqsubset$

Џ Article views: 9

View related articles $₫$ 
Mr. Faraday on Sig. Negro's Magneto-electric Experiments. 45

meet with no sensible resistance from the ethereal medium. Supposing the æther to be constituted like air, and the matter of the earth and planets to consist of very minute spherical atoms, (suppositions, which I have already advanced to explain some phænomena of light*, ) it will follow that no part of the resistance, caused by the condensation of the medium, varies as the simple power of the velocity: also the term $-\frac{r f^{\prime}(t)}{a}$ must be quite insensible. With respect to that part of the motion of the æether which is unaccompanied by change of density, we may say that the velocity of any particle in the same position relatively to the centre of the planet, and to the direction of its motion, has always the same ratio to the velocity of the planet. The consequence of this would be, that the law of the force tending to the sun would not be changed, but its quantity would be diminished. This effect would be accounted for by taking the mass of the planet of less magnitude than it really is, and therefore probably cannot be easily detected by observations. Hence if any resistance be sensible by any change of the orbits or the periodic times, it will depend on the square of the velocity. Also it must be much less sensible in dense bodies like the planets, in which the particles that precede diminish the resistance on those that follow, than in such a rare substance as Encke's comet. This singular body has in fact, in the opinion of competent judges, determined the existence of a medium, or something equivalent, resisting according to the square of the velocity.

Papworth St. Everard, April 17, 1832.

XI. New Experiments relative to the Action of Magnetism on Electro-dynamic Spirals, and a Description of a nerw Electromotive Battery. By Signor Salvatore Dal Negro; with Notes by Michael Faraday, Esq., F.R.S., M.R.I. Corr. Memb. Roy. Acad. Scien. of Paris, \&c. †

[Addressed to Dr. Ambrogio Fusinieri, Director of the Annali Sir, delle Scienze, \&c. \& $c$.]

$\mathrm{O}^{\mathrm{N}}$ repeating the experiments relative to the action of terrestrial magnetism on electro-dynamic spirals, an action which was first observed $\ddagger$ by the two illustrious Italian philo-

* See Phil. Mag. and Annals for March last, p. 161.

4 Communicated by Mr. Faraday.

* This is an error. A long section is devoted to terrestrial magnetoelectric induction in my original researches (140 to 193 ) of the date of 
sophers Nobili and Antinori, it occurred to me to examine the effect of an ordinary magnet on similar spirals at the moment when one of the poles traversed the axis of the spiral (Exp. Res. 39. 41. 114.), and I obtained such results as indicated the path which it would be proper for me to follow, in order to profit by this new property of magnetism. Ultimately I succeeded in constructing a new electrometer, by means of which the efficacy of the instantaneous currents discovered by the celebrated Faraday may be augmented without limit, and obtained in succession with such celerity as to render (as it were) continual the action of these currents*. $\mathrm{He}$ [Dr. Fusinieri] has already witnessed the principal part of these my experiments, and more than once has been so good as to assist me faithfully in registering the results, and has solicited a description that might be made public. I did not hesitate to make a brief exposition that might be transmitted and inserted in the forthcoming number of his Journal. He returned from us as quickly as possible, and did not forget to take with him the magnet I had promised.

$$
\begin{aligned}
& \text { Padua, April 20, 1832. } \\
& \text { Sis most affectionate friend, } \\
& \text { Salvatore Dal NeGro. }
\end{aligned}
$$

\section{Nero Experiments, \&c. \&c.}

1. Place a cylindrical tube of paper surrounded by a spiral of silk-covered copper wire upright upon a little table, and connect the extremities of the spiral with a very sensible galvanometer, constructed according to the method of Signor Nobili : introduce the north pole of an ordinary horse-shoe magnet into the axis of the cylinder, and an electric current will be obtained, which will act strongly on the galvanometer. (Exp. Res. 39. 147.) On withdrawing the pole of the magnet, a current, in the contrary direction, will be obtained (Exp. Res. 39.). On repeating the experiment with the south pole, currents will be manifested in the contrary direction (Exp. Res. 114. \&c.) to those caused by the north pole, and less powerful, as has been observed.

2. Introduce into the same spiral the north pole of a more powerful magnet than the first, and the conflict will produce a much greater effect; I say, " conflict," because the

December 21, 1831. As my brief letter to M. Hachette is continually taken instead of my memoirs as representing my views of magneto-electricity, I venture to add a few notes and references to this paper, in the same man-. ner as I have done to the paper by Signori Nobili and Antinori, at page 401, of the last volume of the Phil. Mag. and Annals.-M. M. F.]

[* I have described at length a different but perfect way of obtaining a continuous current by magneto-electric induction. (Exp. Res. 90.154. 155. 156. \&(.) -M.F.] 
phænomena in question obey the laws of the collisions of solids. The magnetism of rotation discovered by the celebrated Arago has already shown what influence motion has in these phænomena. Then slowly moving the magnet, it may be introduced and removed from the spiral without causing any sensible current. To obtain the maximum effect, it is necessary that the magnetic pole should make its entrance or exit with great velocity. (Exp. Res. 136. 153.258.)

3. Introduce at the same time the poles of the magnet into two equal spirals, having the same direction, and two contrary currents will be obtained, which would destroy each other if the poles of the magnet were of equal strength. But as the north pole is in our latitudes more active than the south, the effect obtained will equal the difference of the two currents, and be in the direction of the greater force; exactly as happens in the collision of solids. It results from this my experiment, that henceforth we may ascertain at once with facility which is the most powerful of two magnets, and how much more active the north pole is than the opposite south pole of the same magnet*.

4. In order to take advantage at the same moment of both the poles of the same magnet, construct two spirals turning in opposite directions, and place them as usual in connection with the galvanometer. Then on introducing the poles of the magnets, an effect will be obtained, equal to the sum of those which could be produced by the poles separately. To measure the effect produced by these two spirals with a more powerful magnet than the first, I was obliged to use a galvanometer of only one-twentieth the sensibility of the first.

5. I immediately perceived that this pair of spirals was a valuable element capable of furnishing a mode of augmenting without limit the efficacy of the instantaneous currents. I therefore instantly constructed a second pair of spirals equal

[* The statement that the north pole is in our latitudes more powerful than the south is a mistake. The cause of the effects obtained by Signor Nrgro will be found at $\mathrm{p} .147$ of my Exp. Research., and is dependent on the inductive force of the earth, as a magnet, upon other magnets, as well as upon soft iron. When a straight magnet is held in the dip, or even vertically with its marked pole downwards, both poles are strengthened; when held with its unmarked pole downwards, both poles are weakeneri. And though when a horse-shoe magnet is held with both poles downwards, as in Signor Negro's experiment, the marked pole is stronger than the unmarked one, it is only because the two limbs are affected as the single magnets just referred to, and the bend of the magnet being the upper part becomes virtually a feeble south pole. If the horse-shoe magnet be held with its poles upwards, then the contrary effect happens, and the unmarked (usually called the south) pole becomes the stronger; or if both poles are in equal relation to the magnetic dip, then both are equally strong.-M.F.] 
to the first, and putting both in connection with the galvanometer, I caused two magnets to enter them contemporaneously, and obtained an effect due to the sum of both pair of spirals. On using still more powerful magnets, even the second galvanometer became useless. The galvanometer which I substituted consists of a rhomboidal needle, about five Paris inches in length, and suspended as in the ordinary compass. The wire which connects the extremities of the spirals passes beneath the needle distant about $3 \frac{1}{2}$ lines, and is parallel to it when the latter is at rest : on obtaining this fortunate result I conceived the idea of constructing a battery of several magnets put in conflict with an equal number of pairs of spirals.

\section{Construction of a new Electro-motive Battery.}

6. I had at command only four magnets, so that for the present I am limited in my construction to four pairs of spirals, as in the manner following: On a little table is placed one after the other four pairs of spirals, with the axes horizontal, and so that the perimeters of the cylinders shall have the same horizontal line as a common tangent, it being parallel to one of the sides of the table. On a second table contiguous to the first, but not in contact, was placed a little carriage consisting of a rectangular table supported on four wheels, by means of which it could easily receive a motion to and fro. The four magnets were placed upon this carriage, so that the poles of each could move horizontally towards the pairs of spirals, and enter within them.

The magnets were firmly fixed on the carriage so as not to alter in position, and the latter was so arranged as to move to and fro only in one direction. On moving the carriage, the limbs of the magnets passed at once into all the spirals, and they could be made to enter or move out with the utmost facility, and with any required velocity.

That the battery thus disposed may give an electric current equal in force to the sum of all the currents excited in the pairs of spirals, it is necessary that all the spirals turning to the right should communicate with each other, that they may form a single metallic wire. The same must be done with all those turning to the left. Then these wires are to be connected in the usual well-known manner with a galvanometer, which we may suppose placed on a third little table, so far distant from the magnets that it may not be influenced by their presence. Although these electric currents are only obtained of instantaneous duration naturally, nevertheless with my battery they may be excited successively with such celerity as to pro- 
duce an action, which is as it were continuous*. From the little I lave done, and from what I have said, it follows that being able by this method to sum up the simultaneous action of an indefinite number of electric currents, this my battery may become fulminating.

I hope I have said enough to enable my readers to comprehend the mode of constructing this electro-motive battery. Hereafter, and by the help of a figure, I will describe the most useful and convenient distribution of the elementary pairs, and the mode of obtaining the maximum effect when employing the smallest possible number of elements, or of pairs of spirals.

Errata relative to Signori Nobili and Antinori's paper. At page 402 of the last Number of Phil. Mag. and Annals, line 23,-for electromo read electrotomo; and in the correspond. ing note, for electronic read electrotonic.

XII. Account of some Experiments in which an Electric Spark was clicited from a natural Magnet. By J Esq. F.R.S. L.\& E. F.G.S.†

THE recent discovery of $\mathrm{Mr}$. Faraday has conclusively demonstrated, that in every case where a magnetic current is created (to use the word current in its ordinary acceptation, as indicative of a peculiar condition, and without reference to any theory whatever), a momentary electric current is induced at right angles to it. 'The experiment may be shown in two ways: either by mechanically causing a magnetic bar to traverse the axis of a helix of copper-wire of considerable length, -or by causing a piece of soft iron, placed in the axis of such a helix, to connect the poles of a horse-shoe magnet, and thus temporarily acquire polarity.

The second method is that which in my late experiments I have entirely employed; and the subject of them has been a very fine natural magnet, capable of supporting $170 \mathrm{lbs}$. presented to the University by Dr. Hope. I willingly avail myself of this opportunity to express my obligations to that gentleman for the numerous and important facilities which have been afforded to my researches, in his laboratory, where the magnet still is.

My preliminary experiments demonstrated, by the action

[* See the note at page 46.-M. F.]

$\uparrow$ Read before the Royal Society of Edinburgh, April 16, 1832, and abridged from the forthcoming volume of their Transactions: See Phil. Mag. and Annals N. S. vol. xi. p. 359.

Third Series. Vol. 1. No. 1. July 1832. 